\title{
Signal sequence-independent membrane targeting of ribosomes containing short nascent peptides within the exit tunnel
}

\author{
Thomas Bornemann $^{1,3}$, Johannes Jöckel ${ }^{1,3}$, Marina V Rodnina ${ }^{2}$ \& Wolfgang Wintermeyer ${ }^{1}$
}

\begin{abstract}
Ribosomes synthesizing inner membrane proteins in Escherichia coli are targeted to the translocon in the plasma membrane by the signal recognition particle (SRP) and the SRP receptor, FtsY. Here we show using a purified system that membrane targeting does not require an exposed signal-anchor sequence, as SRP-dependent targeting takes place with ribosomes containing short nascent peptides, with or without a signal-anchor sequence, within the peptide exit tunnel. Signaling from inside the tunnel involves ribosomal protein L23, which constitutes part of the SRP binding site. When nascent peptides emerge from the ribosome, the targeting complex is maintained with ribosomes exposing a signal-anchor sequence, whereas ribosomes exposing other sequences are released. These results indicate that ribosome-nascent chain complexes containing any nascent peptide within the exit tunnel can enter the SRP targeting pathway to be sorted at the membrane into ribosome-nascent chain complexes that synthesize either membrane or cytosolic proteins.
\end{abstract}

Bacterial plasma membrane proteins are synthesized by ribosomes that are targeted co-translationally to the protein translocation site (translocon) in the membrane by the action of the SRP and the SRP receptor, Fts $\mathrm{Y}^{1-3}$. According to current models ${ }^{4}$, SRP is recruited to ribosome-nascent chain complexes (RNCs) that expose SRP-specific hydrophobic signal-anchor sequences (SASs) outside the peptide exit tunnel, thereby initiating membrane targeting of these RNCs. Quantitative data for the binding of mammalian SRP to wheat RNCs indicate that the affinity is increased substantially when an SAS is exposed on the ribosomes, compared to nontranslating ribosomes ${ }^{5}$. Pertinent quantitative data for the bacterial system are not available. The crystal structure of the translocon suggests how the SAS and subsequent transmembrane helices may laterally exit the translocon and be inserted into the phospholipid bilayer ${ }^{6}$. In eukaryotes, SRP binding to RNCs arrests translation ${ }^{7-9}$, ensuring that the synthesis of proteins destined for membrane insertion or export does not continue before the RNCs are targeted to the translocon. This way, the potentially harmful misfolding or aggregation of membrane proteins synthesized in the cytosol is avoided or minimized. Escherichia coli SRP lacks an arrest domain, and SRP-mediated stalling of translation has not been observed in in vitro systems from E. coli (ref. 10 and our unpublished data). Thus, it is not clear how misfolding or aggregation of partially synthesized membrane proteins in the cytosol is avoided in bacteria. To help answer this question, we have investigated SRP-FtsY recruitment and SRP-FtsY-dependent membrane targeting of E. coli ribosomes.

\section{RESULTS}

Recruitment of SRP to Lep-RNCs not exposing an SAS

As a model for an inner membrane protein that is co-translationally inserted into the membrane in an SRP-dependent manner, we used leader peptidase (Lep) ${ }^{11}$, which contains an SAS in the N-terminal transmembrane helix followed by a second transmembrane helix (Fig. 1a). We prepared RNCs containing nascent N-terminal segments of Lep of varying length (22 to 94 amino acids) by translating 3 -truncated Lep mRNAs in a translation system consisting of purified components (Methods). Lep-RNCs were obtained at 90\% homogeneity and carried nascent peptides of the expected lengths; representative examples are shown in Figure 1b. As examples for nonmembrane proteins, we prepared RNCs that contained nascent peptides of pre- $\beta$-lactamase (Pre- $\beta$-lac) or $\beta$-lactamase $(\beta$-Lac). Additionally, we studied RNCs carrying nascent chains of E. coli HemK, a cytosolic methyltransferase $\mathrm{e}^{12}$.

Affinities of SRP binding to RNCs were determined by fluorescence equilibrium titrations using fluorescence-labeled SRP. Labeled SRP was prepared by binding the SRP protein Ffh to truncated 4.5S RNA(21-81), comprising nucleotides 21 to 81 , to which the fluorophor Alexa Fluor 555 was attached at the $3^{\prime}$ end $^{13}$. 4.5S RNA(21-81) contains the complete Ffh binding site and binds Ffh with the same affinity as full-length RNA, 30-50 $\mathrm{pM}^{14}$. SRP formed with truncated 4.5S RNA binds to RNCs and FtsY with the same affinity as SRP containing full-length RNA (data not shown), and cells expressing truncated 4.5S RNA are viable ${ }^{15}$.

\footnotetext{
${ }^{1}$ Institute of Molecular Biology, and ${ }^{2}$ Institute of Physical Biochemistry, University of Witten/Herdecke, Stockumer Strasse 10, 55448 Witten, Germany. ${ }^{3}$ These authors
} contributed equally to this work. Correspondence should be addressed to W.W. (winterme@uni-wh.de). 


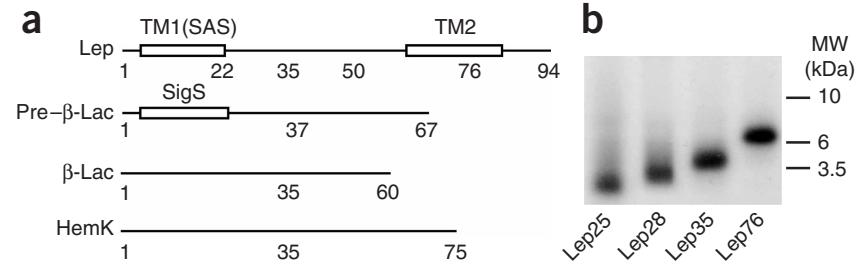

Figure 1 Characterization of RNCs. (a) Schematic of nascent peptides. The lengths of nascent peptides of RNCs used for the experiments are indicated by amino acid positions. The two transmembrane segments of Lep (TM1(SAS) and TM2) and the signal sequence of Pre- $\beta$-Lac (SigS) are indicated by boxes. (b) Gel-electrophoretic analysis of nascent peptides from Lep-RNCs. For details, see Methods.

Initial experiments were conducted with RNCs carrying nascent Lep peptides of 35 or more amino acids. We carried out fluorescence titrations by adding increasing amounts of RNCs to a fixed amount of fluorescence-labeled SRP (Fig. 2a). $K_{\mathrm{d}}$ values of $0.5-1.0 \mathrm{nM}$ were obtained for Lep-RNCs with peptides of 35 or more amino acids. Control titrations yielded $K_{\mathrm{d}}$ values around $50 \mathrm{nM}$ for vacant ribosomes and $70 \mathrm{~S}$ initiation complexes. To determine the minimum length of the peptide that elicits high-affinity binding of SRP, we tested Lep-RNCs with peptide lengths from 22 to 32 amino acids. The affinity of SRP binding remained low, at the level observed for vacant ribosomes, up to Lep peptides of 25 amino acids. At a length of 27 amino acids the affinity started to increase and reached about $1 \mathrm{nM}$ at a chain length of 32 amino acids (Fig. 2b). The affinity remained around $1 \mathrm{nM}$ with ribosomes carrying longer Lep peptides and exposing the SAS.

The peptide exit tunnel of the large ribosomal subunit has a length of $\sim 100 \AA$ from the peptidyl transferase center to the exit ${ }^{16}$. The tunnel would accommodate a peptide of about 28 amino acids in its fully extended conformation, that is, $\sim 3.5 \AA$ per residue, consistent with cross-linking data ${ }^{17}$; longer peptides with more compact conformations may also be accommodated ${ }^{18,19}$. Specifically, it has been shown for Lep-RNCs that a cross-linker at position 37 from the peptidyl transferase center forms a cross-link to Ffh in SRP, whereas at position 35 it does $\operatorname{not}^{17}$. On the basis of these data, in Lep32-RNC, and probably also in Lep35-RNC, the SAS, which starts at amino acid 4 from the $\mathrm{N}$ terminus, is contained within the peptide exit tunnel and is not available for binding SRP.

\section{Recruitment of SRP to RNCs with short nascent peptides}

The results obtained with Lep35-RNC imply that the presence of the peptide within the tunnel is signaled to the SRP binding site outside the tunnel. To examine whether signaling required an SAS, we studied SRP binding to RNCs carrying different peptides within the exit tunnel (Table 1). Replacement of hydrophobic amino acids at positions 10,11 or 18 of the nascent Lep peptide with proline residues, which impairs SAS function (refs. 20,21 and this work), did not interfere with high-affinity binding of SRP when the peptide was contained within the tunnel, whereas the same replacements in exposed peptides strongly impaired SRP binding (Table 1). We also observed high-affinity SRP binding with RNCs carrying the first 37 amino acids of Pre- $\beta$-Lac, which is secreted post-translationally and has a signal sequence that is not recognized by $\mathrm{SRP}^{22}$, with RNCs carrying the first 35 amino acids of mature $\beta$-Lac lacking any signal sequence, or with RNCs carrying $35 \mathrm{~N}$-terminal amino acids of HemK (Table 1). These results demonstrate that high-affinity binding of SRP to RNCs is promoted by nascent peptides of
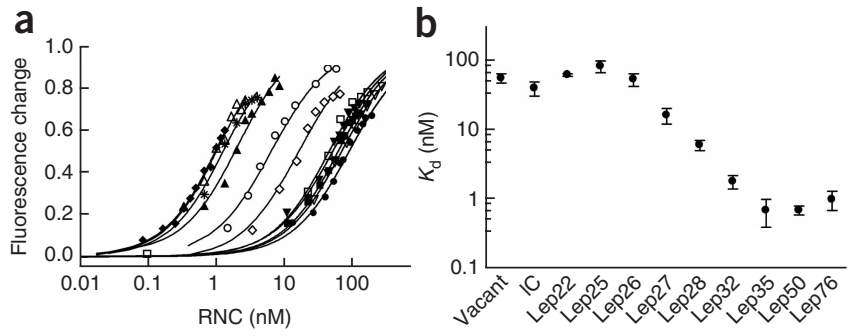

Figure 2 Affinity of SRP for RNCs with varying nascent peptides. (a) Fluorescence titrations. The fluorescence of $0.5 \mathrm{nM} \mathrm{SRP(AIx81)} \mathrm{was}$ monitored upon addition of increasing amounts of Lep-RNCs (Methods). The fluorescence increase (about $10 \%$ maximum) is normalized to 1.0 . The nascent Lep polypeptide had lengths of $22(\nabla), 25(\bullet), 26(\boldsymbol{\nabla}), 27(\diamond)$, $28(\bigcirc), 32(\Delta), 35(\triangle), 50(\diamond)$ or $76\left(^{*}\right)$ amino acids. Titrations were also performed with vacant ribosomes $(\boldsymbol{\square})$ and $70 \mathrm{~S}$ initiation complexes ( $\square$ ). Smooth lines represent fits yielding $K_{d}$ values depicted in $\mathbf{b}$. (b) Affinities of SRP binding. $K_{d}$ values are plotted for vacant ribosomes (vacant), $70 S$ initiation complexes (IC) and Lep-RNCs of increasing lengths of nascent peptides, as indicated. Error bars represent s.d. from at least three independent titrations.

different sequences contained within the exit tunnel and does not require a functional SRP-specific SAS.

Titrations with RNCs exposing longer peptides outside the ribosome revealed that the SRP efficiently discriminates between RNCs that expose an SRP-specific SAS and those that do not (Table 1). Proline at positions 10 and 18 of the Lep76-RNC lowered the affinity of SRP binding to the level of nontranslating ribosomes, presumably owing to impaired SAS binding to SRP, and a similar affinity was observed for $\beta$-Lac60-RNC. The insertion of proline at position 11 of Lep76-RNC, the presence of $67 \mathrm{~N}$-terminal amino acids of Pre- $\beta$-Lac, or the presence of $75 \mathrm{~N}$-terminal amino acids of HemK lowered the affinity to the extent that titrations could not be saturated at the highest concentrations attainable with our RNC preparations. This indicates that in these cases $K_{\mathrm{d}}$ values were $>200 \mathrm{nM}$, much weaker than SRP binding to nontranslating ribosomes. An explanation for the weak binding may be that the polypeptide emerging from the ribosome interferes with SRP binding, perhaps by steric hindrance, which may be influenced by the polypeptide's structure, unless it contains an SAS that strongly binds to SRP.

Table 1 Affinity of SRP for RNCs with short and long nascent peptides $\left(25^{\circ} \mathrm{C}\right)$

\begin{tabular}{|c|c|c|}
\hline \multirow[b]{2}{*}{ Nascent peptide in RNC } & \multicolumn{2}{|c|}{$K_{\mathrm{d}}(\mathrm{nM})$} \\
\hline & Short & Long \\
\hline Lep $^{a}$ & $0.7 \pm 0.1^{f}$ & $1.0 \pm 0.3$ \\
\hline Lep(V1OP) $)^{b}$ & $0.5 \pm 0.2$ & $34 \pm 10$ \\
\hline Lep $(I 11 P)^{b}$ & $2.0 \pm 0.3$ & $>200$ \\
\hline Lep $(I 18 P)^{b}$ & $1.0 \pm 0.5$ & $40 \pm 14$ \\
\hline Pre- $\beta-L a c^{c}$ & $0.8 \pm 0.2$ & $>200$ \\
\hline$\beta$-Lac ${ }^{d}$ & $0.9 \pm 0.4$ & $24 \pm 4$ \\
\hline $\mathrm{HemK}^{\mathrm{e}}$ & $0.4 \pm 0.1$ & $>200$ \\
\hline
\end{tabular}

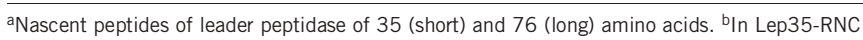
and Lep76-RNC, valine or isoleucine in the SAS were exchanged with proline at the indicated positions. 'Pre- $\beta$-Lac, nascent peptides of pre- $\beta$-lactamase (37 and 67 amino acids long, respectively). ${ }^{\alpha} \beta$-Lac, nascent peptides of mature $\beta$-lactamase without the export signal sequence ( 35 and 60 amino acids long, respectively). ${ }^{\mathrm{H}} \mathrm{HemK}$, nascent peptides of HemK (35 and 75 amino acids long, respectively). ${ }^{\mathrm{f} E r r o r s}$ represent s.d. 

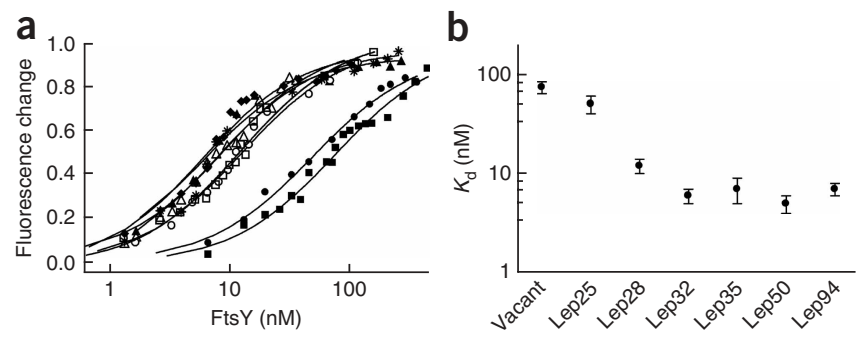

Figure 3 Affinity of FtsY for SRP complexes with Lep-RNCs. (a) Fluorescence titrations. Increasing amounts of FtsY(NG) were added to $1 \mathrm{nM} \mathrm{SRP(Alx81)}$ and $50 \mathrm{nM}$ Lep-RNCs (200 nM for the weaker RNC-SRP complexes with $K_{\mathrm{d}}>10 \mathrm{nM}$ or vacant ribosomes), Lep-RNCs with nascent peptides of $25(\bullet), 28(\bigcirc), 32(\Delta), 35(\triangle), 50(\diamond)$ and $94\left({ }^{*}\right)$ amino acids or vacant ribosomes ( $\mathbf{\square})$ were used. A control titration with full-length Fts $Y$ and Lep50-RNC ( $\square$ ) is also shown. The fluorescence increase (about $10 \%$ maximum) is normalized to 1.0. Smooth lines represent fits with the $K_{\mathrm{d}}$ values depicted in $\mathbf{b}$. (b) $K_{\mathrm{d}}$ values of Fts $\mathrm{Y}(\mathrm{NG})$ binding to SRP bound to Lep-RNCs with varying chain lengths of nascent peptides, as indicated. Error bars represent s.d. from at least three independent titrations.

\section{Enhanced binding of FtsY to high-affinity RNC-SRP complexes}

The fluorescence of ribosome-bound SRP containing labeled 4.5S RNA(21-81) was further increased by the binding of FtsY, allowing us to determine the affinity of FtsY for SRP bound to ribosomes or RNCs. Initially, full-length FtsY and FtsY(NG), which lacks the $\mathrm{N}$-terminal A domain but is fully functional as an SRP receptor ${ }^{23}$, were compared in titrations with SRP bound to Lep50-RNC, yielding identical affinities (Fig. 3a). Thus, titrations with the other Lep-RNCs were performed with Fts $\mathrm{Y}(\mathrm{NG})$, which is functionally equivalent to full-length FtsY and easier to maintain at the high concentrations required for the titrations (Fig. 3a).

The affinity of the FtsY-SRP complex, which is in the order of $50 \mathrm{nM}^{24,25}$, showed little increase when SRP was bound to vacant or initiated ribosomes or to Lep25-RNC. However, the affinity increased with the chain length of the nascent Lep peptide, starting with 28 amino acids, and reached about $6 \mathrm{nM}$ at a chain length of 32 and longer (Fig. 3b). Thus, enhanced binding of FtsY to SRP correlates with increased affinity of SRP binding to RNCs. This result suggests that SRP in the high-affinity ribosome complexes assumes a conformation that has a higher propensity to bind FtsY, in accordance with structural data obtained by cryo- $\mathrm{EM}^{26}$ and fluorescence measurements (I. Buskiewicz, J.J., M.V.R. and W.W., unpublished data), which indicate that the NG domain of the SRP component Ffh is more exposed in those complexes.

\section{Membrane binding of the targeting complex}

To verify that RNCs with short nascent peptides can enter the membrane-targeting pathway, we examined the binding of RNCs to inverted inner membrane vesicles (INVs) from E. coli (Methods), which have been shown to contain functional translocons, SRP and FtsY to allow for efficient RNC targeting ${ }^{1}$. Complex formation was assessed by centrifugation through a sucrose step gradient (Methods). In this assay, free RNCs are pelleted, whereas RNCs that are bound to INVs are retained in the membrane fraction at the boundary between low and high sucrose density. Lep35-RNC, $\beta$-Lac35-RNC, HemK35RNC and Lep76-RNC were bound to INVs, whereas Lep25-RNC, $\beta$-Lac60-RNC and HemK75-RNC were not (Fig. 4a); that is, the binding of RNCs to INVs was strictly correlated with high-affinity binding of SRP-FtsY to the RNCs (Figs. $\mathbf{2 b}$ and $\mathbf{3 b}$, and Table 1). Two more nonmembrane E. coli proteins, HNS, a DNA binding protein, and SelA, an enzyme involved in selenocysteine biosynthesis, showed the same behavior; that is, efficient membrane targeting with RNCs carrying nascent peptides of 35 amino acids and no binding to membranes with peptide lengths of 75 amino acids (Fig. 4a).

The binding of RNCs to INVs was dependent on the presence of GDPNP (Fig. 4a), suggesting that the interaction was mediated by SRP and FtsY. In fact, binding of Lep35-RNC to INVs was completely abrogated in the presence of an antibody against Ffh (Fig. 4b), indicating that membrane binding was mediated by SRP.

\section{Signaling from inside the tunnel involves protein L23}

The ribosomal binding site of SRP at the tunnel exit comprises protein L23 (ref. 27), raising the possibility that the affinity increase for SRP binding caused by the presence of a nascent peptide in the exit tunnel is mediated by protein L23. Bacterial L23 contains a loop of 19 partially conserved amino acids (Val62 to Trp80 in E. coli) that reaches into the tunnel over a length of about $20 \AA$ (Fig. 5a,b) and constitutes a potential interaction site for the nascent peptide. Cross-linking results indicate that a length of the nascent Lep peptide of about 22 amino acids is sufficient to reach protein L23 (ref. 17). To see whether the intra-tunnel loop is involved in signaling, we tested the effect of truncating protein L23 by deleting 8 or 11 amino acids of the intra-tunnel loop, yielding L23 $\Delta 8$ and L23 $\Delta 11$ (Fig. 5a). Ribosomes containing the shortened versions of L23 were isolated from E. coli cells that lacked a functional chromosomal gene for protein L23 (ref. 28) and that were transformed with the plasmid coding

a

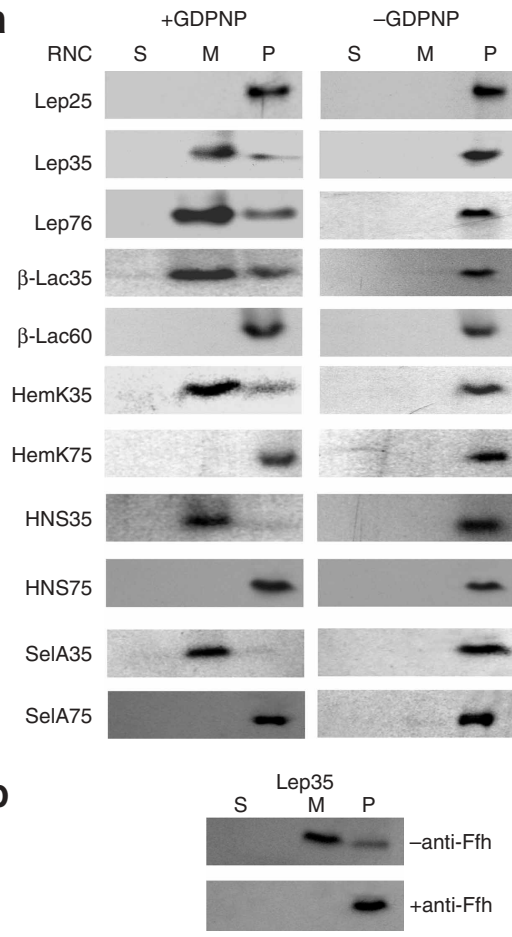

Figure 4 SRP-dependent binding of RNCs to inverted inner membrane vesicles. (a) Dependence on length of nascent peptide. RNCs carrying various nascent peptides (of indicated size) were incubated with INVs, and complexes were analyzed by centrifugation on a sucrose step gradient. Samples of the pellet (P), the membrane fraction $(M)$ and the supernatant (S) were analyzed for nascent peptides by gel electrophoresis (Methods). (b) Effect of SRP depletion of INVs by anti-Ffh antibody. 
a

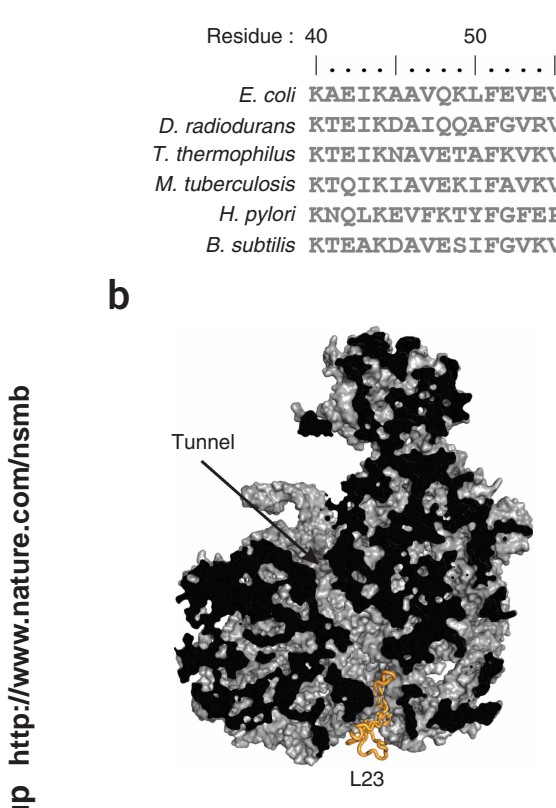

$|\ldots| \ldots|\ldots| \ldots|\ldots| \ldots|\ldots| \ldots|\ldots| \ldots|\ldots| \ldots|\ldots| \ldots|\ldots| \ldots \mid \ldots$

VNTLVVKGKVKRHGQRIGRRSDWRKAYVTLKEGQN-LDFV D. radiodurans KTEIKDAIOOAFGVRVIGISTMNVPGKRKRVGRFIGORNDRKKAIVRLAEGOS-IEAI T. thermophilus KTEIKNAVETAFKVKVVKVNTLHVRGKKKRLGRYLGKRPDRKKAIVQVAPGQK-IEAL tuberculosis KTQIKIAVEKIFAVKVASVNTANRQGKRKRTRTGYGKRKSTKRAIVTLAPGSRPIDIF H. pylori KNQLKEVEKTYFGFEPLKINSLROEGKVKRFRGKLGQRKSEKKFYVKVPEGAS-IAAT B. subtilis RTEARDAVESIFGVKVDKVNIMNYKGKSKRVGRYTGMTSRRRKAIVKLTADSKEIEIF

c

d

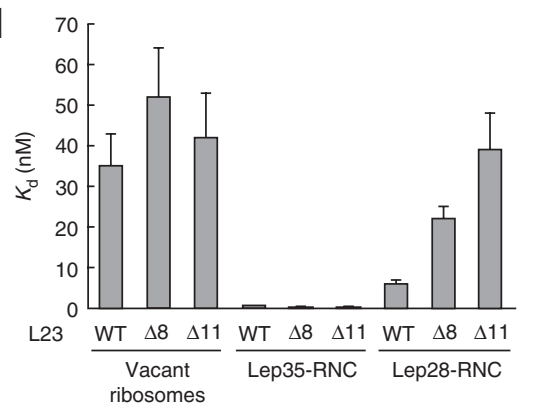

Figure 5 Ribosomal protein L23 and SRP recruitment. (a) Partial sequence alignment of bacterial L23 proteins from Escherichia coli, Deinococcus radiodurans, Thermus thermophilus, Mycobacterium tuberculosis, Helicobacter pylori and Bacillus subtilis. The 11 amino acids in the loop reaching into the ribosomal peptide exit tunnel are in bold, and the deletions of 8 or 11 amino acids are indicated by brackets. (b) The ribosomal exit tunnel. Cut-away structure of the $50 S$ ribosomal subunit from E. coli (PDB entry 1VS6). Protein L23 at the tunnel exit is indicated in yellow. (c) Immunoblot analysis of protein L23 from wild-type (WT) and mutant $(\Delta 8, \Delta 11)$ ribosomes. (d) Affinities of SRP binding to ribosomes or RNCs. $K_{d}$ values of SRP binding to vacant ribosomes or RNCs containing WT or mutant $(\Delta 8, \Delta 11)$ L23 were determined by fluorescence titration as in Figure 2.

that is, strong (nanomolar) binding of the SRP to RNCs that contain a nascent peptide within the peptide exit tunnel of the ribosome and that do not expose a signal sequence at the outside of the ribosome. The same strong effect was observed with several nascent pep-

for L23 $\Delta 8$ or L23 $\Delta 11$, respectively (Methods). The mutations did not impair cell growth under normal growth conditions (data not shown). Ribosomes isolated from these cells contained the respective truncated protein L23, as verified by immunoblotting (Fig. 5c), and were as active as wild-type ribosomes in binding aminoacyl-tRNA and translating Lep-mRNAs (data not shown).

SRP binding to vacant ribosomes or Lep35-RNCs was not affected by the truncations of protein L23 (Fig. 5d). However, SRP binding to Lep28-RNCs containing truncated L23 was six-fold $(\Delta 8)$ or ten-fold $(\Delta 11)$ weaker than to Lep28-RNCs containing wild-type L23 (Fig. 5d). Thus, truncations of the loop of L23 that reaches into the tunnel reduced the affinity of SRP binding to Lep28-RNCs to the level at which SRP binds to vacant ribosomes, suggesting that a nascent peptide of 28 amino acids just reaches the intra-tunnel loop of L23. These results indicate that an interaction of the growing peptide with L23, either at the loop reaching into the tunnel or at the body of the protein at the tunnel exit, alters the protein, perhaps through a conformational change, in such a way as to increase the affinity of SRP binding to the ribosome.

\section{DISCUSSION}

The affinity of E. coli SRP to RNCs exposing the uncleaved signal sequence of Lep, as determined by fluorescence titrations at equilibrium, was found to be $\sim 1.0 \mathrm{nM}$. Higher affinities, around $0.1 \mathrm{nM}$, were reported for the binding of eukaryotic SRPs to RNCs exposing different signal sequences ${ }^{5}$. The difference may reflect the fact that E. coli SRP lacks the elongation-arrest domain of eukaryotic SRP, which, according to cryo-EM ${ }^{26}$, is involved in extensive interactions with the ribosome. The binding of SRP to vacant ribosomes seems to be similar in E. coli and eukaryotes, as the respective affinities, $70 \mathrm{nM}$ (this work) and $80 \mathrm{nM}^{5}$, are about the same. A ten-fold higher affinity was observed for mammalian SRP binding to an RNC that carried a transmembrane segment within the peptide exit tunnel ${ }^{5}$, consistent with the observation of stronger SRP binding to translating ribosomes compared to nontranslating ones in the yeast system ${ }^{29}$. The present results show a similar, although quantitatively larger, effect for E. coli; tides of 35 amino acids in length with different sequences, including Lep signal sequences with proline substitutions, which are not recognized by the SRP on RNCs exposing these sequences. Thus, the effect seems to be independent of the sequence and the propensity for secondary structure formation of the peptide contained within the tunnel.

The last result also pertains to the question of whether translationarrested RNCs, as used here, can be compared to translating ribosomes. The question is whether nascent peptides within the ribosomal exit tunnel of stalled ribosomes can assume a conformation that they would not assume during ongoing translation. Given that translation is much slower (50-100 ms per amino acid incorporated) than secondary structure formation in peptides (micro- or submicroseconds timescale; for review, see ref. 30), there is ample time for nascent peptides to assume a stable secondary structure within the tunnel, even on translating ribosomes. Thus, translating and stalled ribosomes are probably not appreciably different with regard to the structure of the nascent peptide contained in the tunnel. The finding that RNCs containing different nascent peptides show the same high affinity for SRP binding supports this contention.

The present results indicate that a conformational change in the tunnel that is induced by the presence of a nascent peptide, which does not need to contain a signal sequence, is signaled to the SRP binding site at the exit to enhance SRP binding. Notably, the affinity is not increased when the Lep signal sequence is exposed on the ribosome, although, on the basis of the data obtained with the LamB signal sequence ${ }^{31,32}$, the signal peptide is expected to bind to SRP with micromolar affinity. Thus, the free energies of SRP binding to translating ribosomes and to the exposed signal peptide are not additive, indicating that a substantial part of the free binding energy is consumed by a conformational change of the complex, presumably of the SRP, induced by the interaction of the signal sequence with $\mathrm{Ffh}^{32}$. Qualitatively similar observations have been reported for the eukaryotic system, in that the exposure of the signal sequence enhanced SRP binding to a much lesser extent than expected from an additional interaction with micromolar affinity ${ }^{5}$. 
There are several examples of signaling from inside the peptide exit tunnel to functional centers of the ribosome, including elongation arrest by a specific sequence in the SecM protein ${ }^{33,34}$ or the inhibition of termination by the nascent TnaC leader peptide ${ }^{35}$. Signaling from inside the tunnel to the peptide-exit region has been reported for eukaryotic ribosomes, as the interaction of the translating ribosome with the translocon ${ }^{36}$ and the binding of $\mathrm{SRP}^{5}$ were influenced by a nascent transmembrane segment residing in the tunnel. The present results demonstrate a mechanism of signaling from inside the tunnel to the peptide exit for bacterial ribosomes that does not require an SAS. The observation that the maximum affinity for SRP is induced by nascent peptides of $\sim 30$ amino acids, which can reach protein L23 at the tunnel exit ${ }^{17}$, suggests that a contact with protein L23, which reaches into the tunnel and forms part of the SRP binding site ${ }^{27,37}$, is crucial for the effect. The finding that truncations of the intra-tunnel loop of L23 diminish the affinity of SRP binding to RNCs with nascent chains of 28 amino acids strongly supports this contention, although interactions of the nascent peptide with other residues forming the tunnel may be involved as well. Presumably, the interactions of the nascent peptide with protein L23 are nonspecific. Thus, any peptide in the vicinity of L23 - a shorter peptide at the intra-tunnel loop of the bacterial protein or a longer peptide at the globular part of L23 at the end of the tunnel-seems sufficient to induce the conformation of L23 that binds SRP with high affinity. In rapidly translating ribosomes, the time delay between the early contact of the growing peptide with the loop of L23 reaching into the tunnel and with the body of L23 is in the range of $0.5 \mathrm{~s}$, which represents the delay of signaling to the SRP binding site caused by the truncation of L23. This short delay may explain the observation that truncations of the intra-tunnel loop do not impair cell growth, at least not under normal growth conditions, although this might be different under conditions of stress. It should also be noted that archaeal and eukaryotic L23 proteins lack the intratunnel loop.

In the cell, there is much less SRP than ribosomes, with estimates ranging from $25 \%{ }^{38}$ to about $1 \%{ }^{39}$. RNCs with nascent peptides of about 30 amino acids represent a small fraction (probably less than $5 \%$ ) of all translating ribosomes in the cell. This indicates that RNCs with short nascent peptides and SRP are present in the cell in comparable amounts, although SRP may be limiting. Given the $>100$-fold higher affinity for SRP binding (1 nM) of RNCs with short nascent peptides relative to RNCs with long non-SAS peptides (>200 nM), the SRP will bind preferentially to the RNCs with short nascent peptides at equilibrium. The present results were obtained with RNCs prepared from purified components and inverted vesicles isolated from cells. In view of the large affinity differences, it seems likely that indiscriminate recruitment of SRP to most ribosomes early in translation and subsequent targeting to the membrane takes place in the cell as well. In vivo results confirming this contention will be needed in future.

What would be the advantage of such a mechanism for the cell? We propose that initial membrane targeting of translating ribosomes independent of the presence of a signal sequence may alleviate the potential problem caused by the lack of elongation arrest in E. coli. One may argue that, given the small size of bacterial cells, RNCs move to the plasma membrane rapidly enough to obviate the necessity of arresting elongation. However, the bacterial cytosol is crowded with macromolecules, giving rise to slow, 'subdiffusive' movement of particles the size of ribosomes ${ }^{40}$. Given a translation rate of $10-20 \mathrm{~s}^{-1}$, the growing peptide is extended by tens of amino acids within a few seconds, allowing for (mis-)folding of the growing polypeptide before targeting. Thus, by early indiscriminate membrane targeting of translating ribosomes, the time required for the nascent peptide to grow from about 30 amino acids (fully contained in the exit tunnel) to 50 amino acids (20 amino acids exposed outside the tunnel), about $1-2 \mathrm{~s}$, is gained for movement to the membrane. This may increase the probability of proper targeting of RNCs translating membrane proteins, compensating, at least in part, for the lack of elongation arrest and helping to keep the amount of misfolded or aggregated membrane proteins in the cytosol at a level low enough for the protein-degradation systems of the cell to cope with.

\section{METHODS}

Materials. We used the E. coli strain BL21 (DE3) pLysS for the expression of FtsY and Ffh from plasmids pET9-FtsY and pET24-Ffh, respectively ${ }^{41}$, and plasmid pT7-4.5S for the transcription of 4.5S RNA. Plasmids pBCKSBla2, pBCKSBla3 and pBSIIKS-Lep, the last provided by J. Luirink (Free University of Amsterdam), served as templates for mRNA transcription. The plasmid pT $\Delta 198$ encoding FtsY(NG) was a gift from E. Bibi (Weizmann Institute of Science, Rehovot, Israel). We prepared inverted inner membrane vesicles from E. coli MRE600 (refs. 42,43), and we expressed and purified Ffh, FtsY and FtsY(NG) as described ${ }^{13}$. We prepared 4.5S RNA(21-81) and mRNA constructs as described ${ }^{14}$.

For the expression of ribosomes carrying mutant L23, we used the E. coli strain MC4100, in which the chromosomal gene for L23 was deleted ${ }^{28}$. As L23 is essential for viability, we introduced plasmids coding for mutant L23 by exchanging the plasmid coding for wild-type L23, pTrc99B, with the plasmid, derived from the $\mathrm{pCDF}$ duet vector, coding for the respective mutant L23 (ref. 28). Mutations were verified by DNA sequencing. We isolated ribosomes containing wild-type or mutant L23 as described ${ }^{44}$.

RNCs. To prepare RNCs, we translated 3'-truncated mRNAs in a translation system consisting of purified components ${ }^{27,45}$, using purified initiation complexes with $\mathrm{f}\left[{ }^{3} \mathrm{H}\right]$ Met-tRNA ${ }^{\mathrm{fMet}}$, a purified mixture of aminoacyl-tRNA including $\left[{ }^{14} \mathrm{C}\right]$ Leu-tRNA, EF-Tu, EF-Ts, EF-G and GTP. The mRNAs used coded for the first 22 to 94 amino acids of Lep, the first 37 or 67 amino acids of Pre- $\beta$-lac, the first 35 or 60 amino acids of a $\beta$-Lac construct lacking the signal sequence $(\beta-\mathrm{Lac})$, or the first 35 or 75 amino acids of HemK, HNS and SelA. We purified RNCs by centrifugation through a sucrose cushion (1.1 M sucrose in $50 \mathrm{mM}$ Tris- $\mathrm{HCl}, \mathrm{pH} 7.5,70 \mathrm{mM}$ ammonium acetate, $30 \mathrm{mM}$ potassium acetate, $20 \mathrm{mM}$ magnesium acetate). We determined the fraction of ribosomes carrying nascent chains by the amount of $\left[{ }^{3} \mathrm{H}\right]$ Met in ribosome-bound peptides relative to total ribosomes, measured by absorbance at $260 \mathrm{~nm}$. RNCs carrying nascent Lep peptides used in this work were $\sim 90 \%$ homogeneous, the other RNCs $\sim 70 \%$. The length of nascent peptides was estimated from the ratio of $\left[{ }^{14} \mathrm{C}\right]$ Leu to $\mathrm{f}\left[{ }^{3} \mathrm{H}\right]$ Met or determined by gel electrophoresis.

Fluorescence titrations. We prepared 4.5S RNA(Alx81) by labeling the $3^{\prime}$ end of 4.5S RNA(21-81) with Alexa Fluor 555 (ref. 13), and measured fluorescence emission at $565 \mathrm{~nm}$ upon excitation at $515 \mathrm{~nm}$. We performed titrations at $25{ }^{\circ} \mathrm{C}$ in buffer (25 mM HEPES, pH 7.5, $70 \mathrm{mM}$ ammonium acetate, $30 \mathrm{mM}$ potassium acetate, $7 \mathrm{mM}$ magnesium acetate, $10 \%$ glycerol, $0.5 \mathrm{mM}$ GDPNP) by adding small volumes of RNC solution to the solution of fluorescencelabeled SRP $(0.3 \mathrm{ml})$. The added volume did not exceed $5 \%$ of the total volume and the measured signal was corrected for dilution. We performed titrations with FtsY in an analogous way by adding small volumes of FtsY solution to the solution of the respective RNC complex with fluorescence-labeled SRP. The maximum fluorescence increase was about $10 \%$ in both assays.

RNC binding to inverted inner-membrane vesicles. We studied RNC binding to inverted inner-membrane vesicles (INVs) from E. coli as described ${ }^{1}$, except that $10 \mathrm{mM}$ GDPNP- $\mathrm{Mg}^{2+}$ was added to the reaction buffer $(50 \mathrm{mM}$ Trisacetate, $\mathrm{pH} 7.5,70 \mathrm{mM}$ ammonium acetate, $30 \mathrm{mM}$ potassium acetate, $20 \mathrm{mM}$ magnesium acetate). Samples $(0.05 \mathrm{ml})$ were layered on top of a sucrose step gradient of $0.77 \mathrm{M}$ (top) and $1.44 \mathrm{M}$ (bottom) of sucrose (total volume $0.15 \mathrm{ml}$ ) in the same buffer. After $30 \mathrm{~min}$ of centrifugation at $135,000 \times g$ (Sorvall S100AT3 centrifuge), we took $0.08 \mathrm{ml}$ fractions from the supernatant (S), the boundary between low and high sucrose $(\mathrm{M})$ and the redissolved pellet 
(P). After TCA precipitation and hydrolysis of peptidyl-tRNA, samples were analyzed on $16 \%$ Tris-Tricine polyacrylamide gels containing SDS and 2,5-diphenyloxazole ${ }^{46}$. Radiolabeled polypeptides were visualized by fluorography and quantified using ImageQuant software (GE Healthcare).

\section{ACKNOWLEDGMENTS}

We thank E. Bibi, Weizmann Institute of Science, Rehovot, Israel, and J. Luirink, Free University of Amsterdam, for kindly providing plasmid constructs, E. Deuerling, University of Konstanz, Germany, for providing the E. coli strain lacking protein L23, and A. Tonevitsky, Moscow State University, for the antibody against Ffh. We are grateful to M. Wahl, Max-Planck-Institut für Biophysikalische Chemie, Göttingen, Germany, for help with preparing Figure $\mathbf{5 b}$, and to A. Böhm, S. Möbitz, C. Schillings and P. Striebeck for valuable technical assistance. The work was supported by the Deutsche Forschungsgemeinschaft and the Fonds der Chemischen Industrie.

\section{AUTHOR CONTRIBUTIONS}

T.B. and J.J. performed the experiments, M.V.R. and W.W. supervised the work and wrote the manuscript.

Published online at http://www.nature.com/nsmb/

Reprints and permissions information is available online at http://npg.nature.com/ reprintsandpermissions

1. Koch, H.G. et al. In vitro studies with purified components reveal signal recognition particle (SRP) and SecA/SecB as constituents of two independent protein-targeting pathways of Escherichia coli. Mol. Biol. Cell 10, 2163-2173 (1999).

2. Ulbrandt, N.D., Newitt, J.A. \& Bernstein, H.D. The E. coli signal recognition particle is required for the insertion of a subset of inner membrane proteins. Cel/ 88, 187-196 (1997).

3. MacFarlane, J. \& Müller, M. The functional integration of a polytopic membrane protein of Escherichia coli is dependent on the bacterial signal-recognition particle. Eur. J. Biochem. 233, 766-771 (1995).

4. Luirink, J., von Heijne, G., Houben, E. \& de Gier, J.W. Biogenesis of inner membrane proteins in Escherichia coli. Annu. Rev. Microbiol. 59, 329-355 (2005).

5. Flanagan, J.J. et al. Signal recognition particle binds to ribosome-bound signal sequences with fluorescence-detected subnanomolar affinity that does not diminish as the nascent chain lengthens. J. Biol. Chem. 278, 18628-18637 (2003).

6. Van den Berg, B. et al. X-ray structure of a protein-conducting channel. Nature $\mathbf{4 2 7}$, 36-44 (2004).

7. Walter P. \& Blobel, G. Translocation of proteins across the endoplasmic reticulum III. Signal recognition protein (SRP) causes signal sequence-dependent and site-specific arrest of chain elongation that is released by microsomal membranes. J. Cell Biol. 91, 557-561 (1981).

8. Wolin, S.L. \& Walter, P. Signal recognition particle mediates a transient elongation arrest of preprolactin in reticulocyte lysate. J. Cell Biol. 109, 2617-2622 (1989).

9. Mason, N., Ciufo, L.F. \& Brown, J.D. Elongation arrest is a physiologically important function of signal recognition particle. EMBO J. 19, 4164-4174 (2000).

10. Raine, A. et al. Targeting and insertion of heterologous membrane proteins in E. coli. Biochimie 85, 659-668 (2003).

11. de Gier, J.W. et al. Assembly of a cytoplasmic membrane protein in Escherichia coli is dependent on the signal recognition particle. FEBS Lett. 399, 307-309 (1996).

12. Nakahigashi, K. et al. HemK, a class of protein methyl transferase with similarity to DNA methyl transferases, methylates polypeptide chain release factors, and hemK knockout induces defects in translational termination. Proc. Natl. Acad. Sci. USA 99, 1473-1478 (2002).

13. Buskiewicz, I., Kubarenko, A., Peske, F., Rodnina, M.V. \& Wintermeyer, W. Domain rearrangement of SRP protein Ffh upon binding 4.5S RNA and the SRP receptor FtsY. RNA 11, 947-957 (2005).

14. Buskiewicz, I. et al. Conformations of the signal recognition particle protein Ffh from Escherichia coli as determined by FRET. J. Mol. Biol. 351, 417-430 (2005).

15. Batey, R.T., Rambo, R.P., Lucast, L., Rha, B. \& Doudna, J.A. Crystal structure of the ribonucleoprotein core of the signal recognition particle. Science 287, 1232-1239 (2000).

16. Ban, N., Nissen, P., Hansen, J., Moore, P.B. \& Steitz, T.A. The complete atomic structure of the large ribosomal subunit at $2.4 \AA$ resolution. Science $\mathbf{2 8 9}, 905-920$ (2000)

17. Houben, E.N., Zarivach, R., Oudega, B. \& Luirink, J. Early encounters of a nascent membrane protein: specificity and timing of contacts inside and outside the ribosome. J. Cell Biol. 170, 27-35 (2005).
18. Woolhead, C.A., McCormick, P.J. \& Johnson, A.E. Nascent membrane and secretory proteins differ in FRET-detected folding far inside the ribosome and in their exposure to ribosomal proteins. Cell 116, 725-736 (2004).

19. Lu, J. \& Deutsch, C. Folding zones inside the ribosomal exit tunnel. Nat. Struct. Mol. Biol. 12, 1123-1129 (2005).

20. Ryan, P. \& Edwards, C.O. Systematic introduction of proline in a eukaryotic signal sequence suggests asymmetry within the hydrophobic core. J. Biol. Chem. 270, 27876-27879 (1995)

21. Hessa, T. et al. Recognition of transmembrane helices by the endoplasmic reticulum translocon. Nature 433, 377-381 (2005).

22. Beha, D., Deitermann, S., Müller, M. \& Koch, H.G. Export of $\beta$-lactamase is independent of the signal recognition particle. J. Biol. Chem. 278, 22161-22167 (2003).

23. Eitan, A. \& Bibi, E. The core Escherichia coli signal recognition particle receptor contains only the N and G domains of FtsY. J. Bacteriol. 186, 2492-2494 (2004).

24. Jagath, J.R. et al. Important role of the tetraloop region of 4.5S RNA in SRP binding to its receptor FtsY. RNA 7, 293-301 (2001).

25. Peluso, P. et al. Role of $4.5 \mathrm{~S}$ RNA in assembly of the bacterial signal recognition particle with its receptor. Science 288, 1640-1643 (2000).

26. Halic, M. et al. Structure of the signal recognition particle interacting with the elongation-arrested ribosome. Nature 427, 808-814 (2004).

27. Gu, S.Q., Peske, F., Wieden, H.J., Rodnina, M.V. \& Wintermeyer, W. The signa recognition particle binds to protein $\mathrm{L} 23$ at the peptide exit of the Escherichia coli ribosome. RNA 9, 566-573 (2003).

28. Kramer, G. et al. L23 protein functions as a chaperone docking site on the ribosome. Nature 419, 171-174 (2002).

29. Raue, U., Oellerer, S. \& Rospert, S. Association of protein biogenesis factors at the yeast ribosomal tunnel exit is affected by the translational status and nascent polypeptide sequence. J. Biol. Chem. 282, 7809-7816 (2007).

30. Callender, R.H., Dyer, R.B., Gilmanshin, R. \& Woodruff, W.H. Fast events in protein folding: the time evolution of primary processes. Annu. Rev. Phys. Chem. 49, 173-202 (1998).

31. Miller, J.D., Bernstein, H.D. \& Walter, P. Interaction of E. coli Ffh/4.5S ribonucleoprotein and FtsY mimics that of mammalian signal recognition particle and its receptor. Nature 367, 657-659 (1994).

32. Zheng, N. \& Gierasch, L.M. Domain interactions in E. coli SRP: stabilization of M domain by RNA is required for effective signal sequence modulation of NG domain. Mol. Cell 1, 79-87 (1997).

33. Nakatogawa, H. \& Ito, K. The ribosomal exit tunnel functions as a discriminating gate. Cell 108, 629-636 (2002).

34. Mitra, K. et al. Elongation arrest by SecM via a cascade of ribosomal RNA rearrangements. Mol. Cell 22, 533-543 (2006).

35. Cruz-Vera, L.R., Gong, M. \& Yanofsky, C. Changes produced by bound tryptophan in the ribosome peptidyl transferase center in response to TnaC, a nascent leader peptide. Proc. Natl. Acad. Sci. USA 103, 3598-3603 (2006).

36. Liao, S., Lin, J., Do, H. \& Johnson, A.E. Both lumenal and cytosolic gating of the aqueous ER translocon pore are regulated from inside the ribosome during membrane protein integration. Cel/ 90, 31-41 (1997).

37. Ullers, R.S. et al. Interplay of signal recognition particle and trigger factor at L23 near the nascent chain exit site on the Escherichia coli ribosome. J. Cell Biol. 161, 679-684 (2003).

38. Hsu, L.M., Zagorski, J. \& Fournier, M.J. Cloning and sequence analysis of the Escherichia coli 4.5 S RNA gene. J. Mol. Biol. 178, 509-531 (1984).

39. Jensen, C.G. \& Pedersen, S. Concentrations of 4.5S RNA and Ffh protein in Escherichia coli: the stability of Ffh protein is dependent on the concentration of 4.5S RNA. J. Bacteriol. 176, $7148-7154$ (1994).

40. Golding, I. \& Cox, E.C. Physical nature of bacterial cytoplasm. Phys. Rev. Lett. 96 098102 (2006)

41. Jagath, J.R., Rodnina, M.V. \& Wintermeyer, W. Conformational changes in the bacterial SRP receptor FtsY upon binding of guanine nucleotides and SRP. J. Mol. Biol. 295 745-753 (2000).

42. De Vrije, T., Tommassen, J. \& De Kruijff, B. Optimal posttranslational translocation of the precursor of PhoE protein across Escherichia coli membrane vesicles requires both ATP and the protonmotive force. Biochim. Biophys. Acta 900, 63-72 (1987).

43. Müller, M. \& Blobel, G. In vitro translocation of bacterial proteins across the plasma membrane of Escherichia coli. Proc. Natl. Acad. Sci. USA 81, 7421-7425 (1984).

44. Rodnina, M.V. \& Wintermeyer, W. GTP consumption of elongation factor Tu during translation of heteropolymeric mRNAs. Proc. Natl. Acad. Sci. USA 92, 1945-1949 (1995).

45. Gu, S.Q. et al. Conformation of 4.5S RNA in the signal recognition particle and on the 30S ribosomal subunit. RNA 11, 1374-1384 (2005).

46. Laskey, R.A. \& Mills, A.D. Quantitative film detection of ${ }^{3} \mathrm{H}$ and ${ }^{14} \mathrm{C}$ in polyacrylamide gels by fluorography. Eur. J. Biochem. 56, 335-341 (1975). 